\title{
El reto: educar para convivir
}

\section{The challenge: educate to coexist}

\author{
Andrea Carolina Montaña Beltrán* \\ Zulma Carmenza Rocha Adame*** \\ Ana Milena Rojas Hernández****
}

Fecha de recepción: 15 Marzo de 2016

Fecha de aprobación: 2 de diciembre de 2016

Artículo de Reflexión

\section{Resumen}

Educar para convivir es el reto que asumen los maestros de hoy para trabajar en la construcción de una sociedad en paz. Esta investigación tuvo como objetivo desarrollar estrategias pedagógicas que promovieran la convivencia escolar en los estudiantes de los grados tercero, cuarto y quinto de la sede Campoamor, de la Institución Educativa Politécnico Álvaro González Santana (IEPAGS), en la ciudad de Sogamoso - Boyacá. Se definieron tres ejes que le dieron sentido a la investigación: convivencia escolar, competencias ciudadanas comunicativas (CCC), y clima de aula. Así mismo, se implementaron estrategias pedagógicas propuestas desde la Guía 49 "Guías pedagógicas para la convivencia escolar" del Ministerio de Educación Nacional - MEN, dirigidas al desarrollo de competencias ciudadanas comunicativas: argumentación, asertividad y escucha activa.A partir de los resultados encontrados, se realizó una evaluación mediante el análisis a dicha implementación que nos condujo a la construcción de recomendaciones dirigidas a la IEPAGS para fortalecer la convivencia escolar.

Palabras clave: convivencia escolar, competencias ciudadanas, asertividad, argumentación, escucha activa.
*Institución Educativa Politécnico Álvaro González - Boyacá, Colombia ancamobe@hotmail.com **Institución Educativa Politécnico Álvaro González -Boyacá, Colombia zulcaroa@gmail.com ***Institución Educativa Politécnico Álvaro González - Boyacá, Colombia anmilrohe@hotmail.com 


\section{Abstract}

Educating to coexist is the challenge assumed by today's teachers to work in the construction of a peaceful society. This research aimed to develop pedagogical strategies that promote school coexistence in the third, fourth and fifth grade students of the branch "Campoamor" of the "Álvaro González Santana” Educational Institution (IEPAGS), in Sogamoso - Boyacá. school life, communicative citizenship skills (CCC), and classroom climate: three axes that gave meaning to the research were defined. Likewise, pedagogical strategies proposed from
Guide 49 "Pedagogical guidelines for school coexistence" of the Ministry of National Education - MEN, aimed at the development of communicative citizenship skills: argumentation, assertiveness and active listening. Based on the results, an evaluation was made through the analysis of this implementation that led to the construction of recommendations addressed to the IEPAGS to strengthen school coexistence.

Keywords: school coexistence, citizenship skills, assertiveness, argumentation, active listening. 


\section{Introducción}

La convivencia escolar es un eje importante para la consolidación de espacios en el aula, que contribuyen a la construcción de una nueva ciudadanía que responda a los retos que proponen las actuales generaciones; estudiantes que participen con sus actitudes y actuaciones al desarrollo de la paz, que tengan la capacidad de escuchar, de argumentar sus ideas, tener un criterio autónomo distante de las manipulaciones masivas y tener la capacidad de transmitir sus ideas respetando los derechos de sí mismo y del otro.

Desde la Constitución Política de 1991, Colombia comenzó a pensar en un ciudadano que no solo se construyera sino que pensara en colectivo y trabajara por una sociedad democrática, participativa, pluralista, diversa e intercultural. Bajo estos intereses, el Ministerio de Educación Nacional, asumió el liderazgo para orientar el proceso de construcción de convivencia en la escuela a partir del desarrollo de las competencias ciudadanas, dirigidos a la construcción de una nueva ciudadanía. Es así, como dieron origen a la política ministerial 2010-2014 "educación de calidad: camino a la prosperidad". De este programa, se desprende el desarrollo de competencias ciudadanas que se orienta a desarrollar las habilidades, destrezas y conocimientos sobre ciudadanía y convivencia en los estudiantes (MEN, 2013).

Diferentes autores en Colombia han trabajado el desarrollo de competencias ciudadanas, como es el caso de Antanas Mockus, Alexander Ruiz, Enrique
Chaux, entre otros. Chaux, por su parte, ha sido uno de los principales asesores para el MEN en cuanto a la construcción de los estándares sobre competencias ciudadanas, y es uno de los referentes teóricos más valiosos que se identifican en la Guía 49 de esta entidad nacional.

El fortalecimiento de la convivencia escolar ha sido preocupación de la presente investigación, que creemos oportuna y necesaria para la materialización de estos intereses pedagógicos a partir del fortalecimiento de la convivencia escolar,- a la luz de la Guía 49 del Ministerio de Educación Nacional (MEN).

Es así como, desde la práctica pedagógica realizada en el aula, hicimos un análisis profundo mediante la aplicación de diferentes instrumentos como la observación, el diario de campo, talleres y encuestas, mediante las cuales identificamos las dificultades en el ejercicio de las competencias ciudadanas comunicativas. Estas situaciones, infieren en el proceso de aprendizaje afectando el clima de aula y los resultados académicos de los estudiantes.

Teóricamente, en primer lugar, nos basamos en los documentos presentados por el MEN para el desarrollo de competencias ciudadanas: las cartillas orientadoras para la institucionalización de las competencias ciudadanas (brújula); la Guía 6 estándares básicos de competencias ciudadanas y la Guía 49: Guías pedagógicas para la convivencia escolar. Nos basamos en los aportes teóricos de Enrique Chaux, Julio Enrique Soler, Rosario Ortega y Antanas Mockus, quienes han dedicado gran
Desde la Constitución Política de 1991, Colombia comenzó a pensar en un ciudadano que no solo se construyera sino que pensara en colectivo y trabajara por una sociedad democrática, participativa, pluralista, diversa e intercultural. 
El desarrollo de la investigación se sustentó en la Investigación acción (Elliott, 1990), el paradigma socio-crítico, enfoque cualitativo, (Hernández, Fernández y Baptista, 2006), bajo tres fases metodológicas. parte de su vida al desarrollo de teorías y prácticas alrededor de las competencias ciudadanas.

A partir de estas teorías, se trazó el objetivo de fortalecer la convivencia escolar en los estudiantes de los grados tercero, cuarto y quinto de la sede Campoamor, Jornada de la Mañana de la Institución Educativa Politécnico Álvaro González Santana mediante el desarrollo de competencias ciudadanas comunicativas: escucha activa, argumentación y asertividad. Estas se definen como:

Una serie de conocimientos, actitudes y habilidades comunicativas, emocionales, cognitivas e integradoras que funcionan de manera articulada para que todas las personas seamos sujetos sociales activos de derechos, es decir, para que podamos ejercer plenamente la ciudadanía respetando, difundiendo, defendiendo, garantizando y restaurando nuestros derechos. (MEN, 2004, p. 22)

Es así que, el desarrollo de la investigación se sustenta en el paradigma socio - crítico, enfoque cualitativo (Hernández, Fernández y Baptista, 2006), bajo el tipo de investigación acción, (Elliott, 1993). La primera fase realizada fue el diagnóstico, en la segunda fase investigativa se contempló la implementación de las estrategias pedagógicas, con miras a desarrollar las competencias ciudadanas comunicativas. Por último, en la tercera fase, se realizó la evaluación de estas estrategias.

Finalmente, la evaluación de los resultados nos permitió la construcción de una reflexión pedagógica desde la cual se emiten algunas recomendaciones acordes a las necesidades de la población escolar, y en las que se espera que, en un futuro próximo, logren ser articuladas en el Proyecto Educativo Institucional de la Institución y la gestión académica de la Institución para consolidar la articulación y trazabilidad del fortalecimiento de la convivencia escolar, dado que es necesario un proceso constante y con el debido seguimiento para que sea visible y valioso. Además, se considera que puede convertirse en una herramienta significativa para el mejoramiento del ambiente escolar y, por ende, repercutir en el mejoramiento de la calidad educativa.

\section{EL RETO: EDUCAR PARA CONVIVIR}

La IEPAGS cuenta con 3697 estudiantes distribuidos en cuatro sedes de primaria y una de bachillerato. La Sede Campoamor atiende una población de 209 estudiantes en la jornada de la mañana. Se tomó como muestra para el desarrollo de esta investigación, los estudiantes de los grados tercero, cuarto y quinto de esta jornada, durante el año 2016, cada uno de ellos con 36 estudiantes, que en su totalidad suman 108, las edades promedio para estos grados escolares oscilan entre los 8 y 12 años.

El desarrollo de la investigación se sustentó en la Investigación acción (Elliott, 1990), el paradigma sociocrítico, enfoque cualitativo, (Hernández, Fernández y Baptista, 2006), bajo tres fases metodológicas. En la primera fase, se hizo un diagnóstico que permitió 
la identificación de situaciones que generaban dificultades de convivencia escolar en el aula de clase. En la segunda fase, se ejecutó la implementación de las estrategias pedagógicas con miras a desarrollar las competencias ciudadanas comunicativas para el fortalecimiento de la convivencia escolar. Finalmente, en la tercera fase, se realizó la evaluación de estas estrategias, este proceso permitió determinar la manera como las estrategias pedagógicas fortalecieron o no la convivencia escolar.

A partir de la Investigación Acción (Elliott, 1993), se desarrollaron las siguientes fases:

Diagnóstico: mediante esta fase, se logró esclarecer e identificar las situaciones que generaban dificultades de convivencia escolar en el aula escolar. De esta manera, se llegó a un conocimiento real y concreto del contexto. Este sirvió como punto de partida para el desarrollo de las acciones que nos condujeran al fortalecimiento de la convivencia escolar de acuerdo con las necesidades encontradas. En esta fase, fueron utilizados instrumentos como: la observación, definida como "El registro sistemático válido y confiable de comportamientos o conductas manifiestas" (Hernández, Fernández y Baptista, 2006 p. 309); para esta se elaboró y utilizó una "Ficha de Observación" que permitió recoger datos en forma ordenada y objetiva. La ficha de observación que se muestra en la figura 1, permitió hacer un registro sistemático y trazable de los hallazgos encontrados durante el primer periodo escolar del año 2016, comprendido entre el 25 de enero y el 01 de abril; se registraron las observaciones enfocadas en cada una de las tres aulas escolares seleccionadas en la investigación.

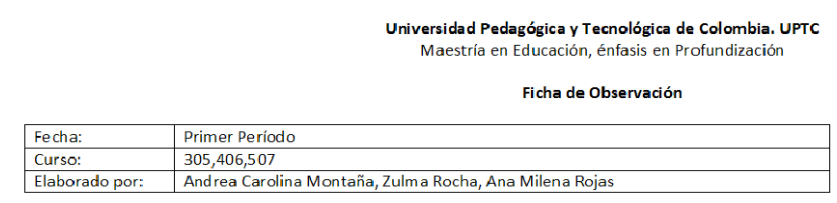

\begin{tabular}{|l|c|c|c|c|c|}
\hline \multicolumn{1}{|c|}{ CRITERIO DE OBSERVACIÓN } & $\begin{array}{c}\text { TOTALMENTE } \\
\text { INAPORPIADO } \\
\%\end{array}$ & $\begin{array}{c}\text { INAPROPIADO } \\
\%\end{array}$ & $\begin{array}{c}\text { MEDIANAMENTE } \\
\text { APROPIADO } \\
\%\end{array}$ & $\begin{array}{c}\text { APROPIADO } \\
\%\end{array}$ & $\begin{array}{c}\text { MUY } \\
\text { APROPIADO } \\
\%\end{array}$ \\
\hline Expresiones de agresión verbal & 5 & 25 & 20 & 40 & 10 \\
\hline Expresiones de agresión fisica & 2 & 5 & 13 & 40 & 40 \\
\hline Interés por el gobierno escolar & 0 & 0 & 10 & 10 & 80 \\
\hline Actos de discriminación & 0 & 2 & 8 & 40 & 50 \\
\hline Actítudes hacia la justificación del uso de violencia & 4 & 26 & 25 & 25 & 20 \\
\hline Actitudes de escucha activa & 70 & 10 & 10 & 5 & 5 \\
\hline Asertividad & 75 & 15 & 5 & 3 & 2 \\
\hline Actitudes de participación & 20 & 10 & 30 & 20 & 20 \\
\hline Cumplimiento de normas & 15 & 15 & 20 & 30 & 20 \\
\hline Capacidad de argumentación & 65 & 15 & 10 & 5 & 5 \\
\hline Empatía hacia la convivencia & 30 & 10 & 10 & 30 & 20 \\
\hline Capacidad para manejar la rabia & 25 & 15 & 15 & 15 & \\
\hline Capacidad para transmitir ideas & 40 & 20 & 15 & 15 & 10 \\
\hline
\end{tabular}

Criterios establecidos de acuerdo a la Gú́ N 49 del MEN Gúas pedagógicas para la convivencia escolar.

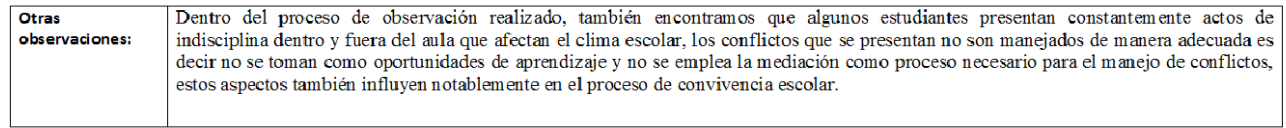

Figura 1: Ficha de observación

Fuente: elaboración propia. 
De igual manera, se llevó el diario de campo entendido como un instrumento de registro (ver figura 2) que permitió sistematizar experiencias, para luego desarrollar el análisis de resultados. Según Hernández, Fernández y Baptista (2006), "es muy necesario llevar registros y elaborar anotaciones durante los eventos o sucesos vinculados al proyecto" (p. 541). Este instrumento proporcionó una aproximación contundente de las características propias del aula escolar, los comportamientos inherentes de cada uno de los estudiantes y logró otorgar una mejor comprensión de lo que ocurre al interior del aula de clase.

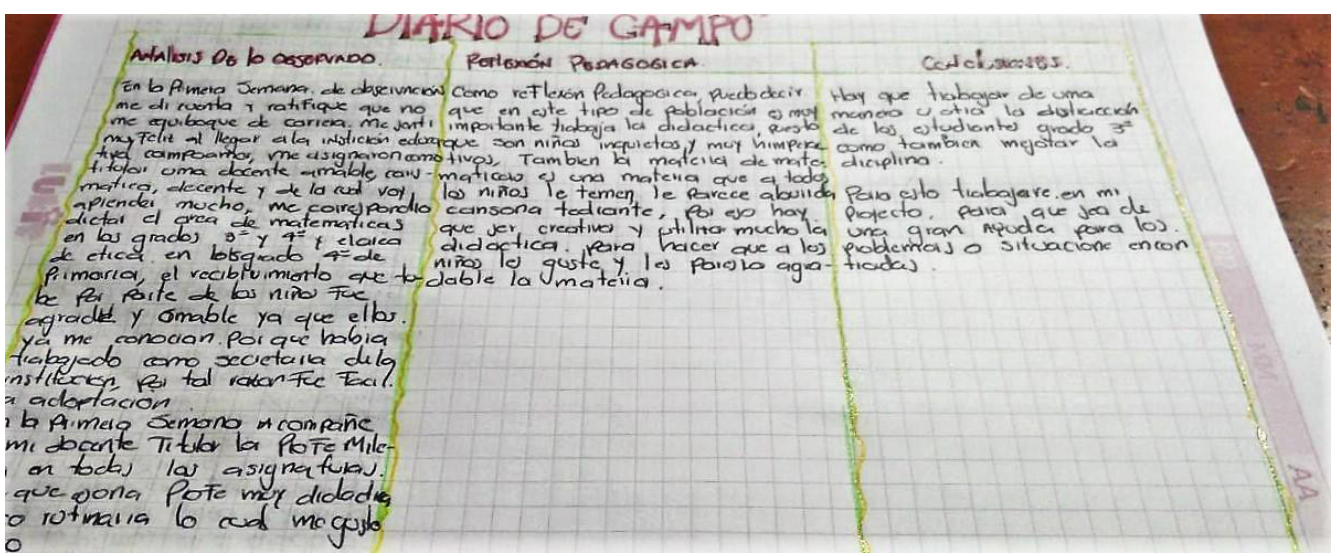

Figura 2. Diario de campo Fuente: elaboración propia.

Otro de los instrumentos fue la "Encuesta Institucional" (figura 3), que arrojó resultados importantes para identificar y conocer el contexto familiar de los estudiantes; "El método de encuesta es un cuestionario estructurado que se da a una muestra de la población y está diseñado para obtener información específica" (Bonilla y Rodríguez, 2005 p. 135). La tabulación y posterior análisis de estos datos, permitieron aproximaciones claras a la caracterización de la población. En la figura 3, se observa la Encuesta Institucional implementada y analizada:

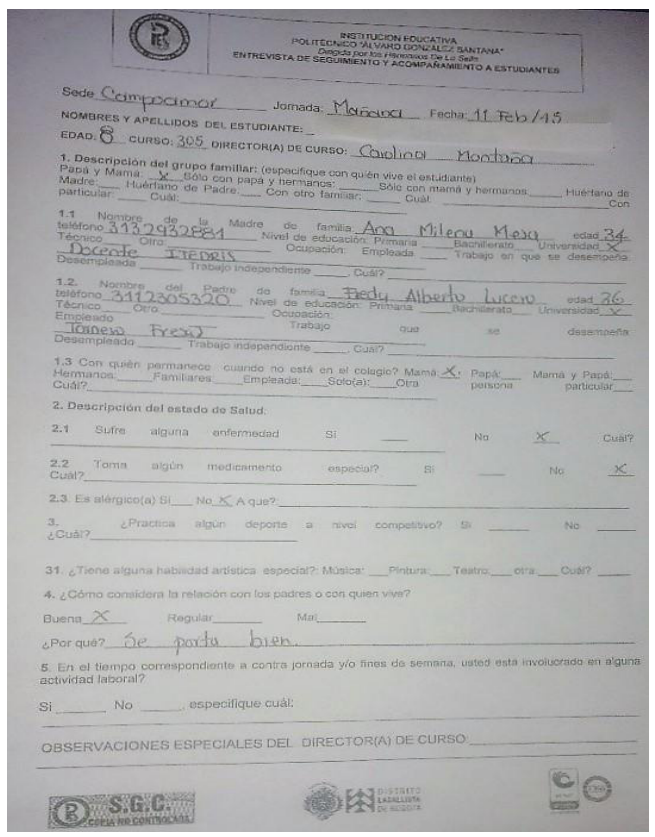

Figura 3. Encuesta institucional. Fuente: IEPAGS, 2016. 
De igual manera, se implementó la "Encuesta a Padres de Familia", instrumento mediante el que se logró identificar acciones concretas referentes al manejo de conflictos y situaciones cotidianas de convivencia en el hogar. Se obtuvo la siguiente información que se muestra en la figura 4: un 72.2 $\%$ se abstiene de usar el castigo físico y un $53.7 \%$ no toleran el abuso sexual en ninguna de sus manifestaciones, es decir, que como padres de familia en su mayoría utilizan formas menos violentas de castigo hacia sus hijos.

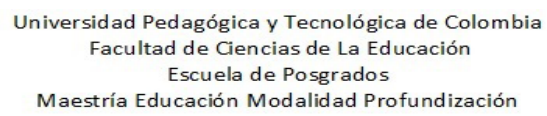

Apreciados padres de familia:

A continuación se presenta una lista de acciones que las familias pueden llevar a cabo para promover el desarrollo de las competencias ciudadanas. (Chaux, et al., 2013). Les pedimos responder la siguiente encuesta con la mayor franqueza posible. Al hacerlo, nos ayudarán con mayor precisión a la identificación de estrategias que mejoren la convivencia escolar en la sede Campoamor.

Marque con una X la opción más acorde a su familia:

\begin{tabular}{|c|c|c|}
\hline ACCIONES QUE LLEVAMOS A CABO EN LA FAMILIA & SI & NO \\
\hline \multicolumn{3}{|l|}{ EN EL TRATO CON LAS NIÑAS Y LOS NIÑOS } \\
\hline \multicolumn{3}{|l|}{ Me abstengo en todos los casos de usar el castigo físico } \\
\hline \multicolumn{3}{|l|}{ No tolero el abuso sexual en ninguna de sus manifestaciones } \\
\hline \multicolumn{3}{|l|}{$\begin{array}{l}\text { Promovemos que los conflictos en las familias se manejen de manera pacífica, con actitud } \\
\text { de escucha, tomando en cuenta las distintas versiones y perspectivas de lo ocurrido, y } \\
\text { buscando alternativas que favorezcan los intereses de todas las personas }\end{array}$} \\
\hline \multicolumn{3}{|l|}{$\begin{array}{l}\text { Promovemos que todas las personas que conforman la familia puedan expresar lo que } \\
\text { sienten sin herir a otras personas }\end{array}$} \\
\hline \multicolumn{3}{|l|}{$\begin{array}{l}\text { Promovemos que todas las personas que conforman las familias nos preocupemos por el } \\
\text { bienestar de las demás personas }\end{array}$} \\
\hline \multicolumn{3}{|l|}{$\begin{array}{l}\text { Aprovechamos oportunidades para promover las prácticas de convivencias ciudadanas } \\
\text { como por ejemplo, escuchar, identificar las emociones propias y las de otras personas, } \\
\text { empatía, argumentar puntos de vista, etc. }\end{array}$} \\
\hline \multicolumn{3}{|l|}{$\begin{array}{l}\text { Promovemos la capacidad para ponerse en los zapatos de otras personas y entender cómo } \\
\text { se sienten }\end{array}$} \\
\hline \multicolumn{3}{|l|}{$\begin{array}{l}\text { Existen en las familias normas que definen claramente lo que no está permitido. Es claro } \\
\text { que no está permitido hacerle daño a otras personas, a sí mismas o sí mismos. }\end{array}$} \\
\hline \multicolumn{3}{|l|}{$\begin{array}{l}\text { Verifico los recursos que tienen las niñas, niños y adolescentes en casa para solucionar } \\
\text { conflictos y contribuyo a fortalecer las habilidades que tiene y a desarrollar las que no } \\
\text { poseen }\end{array}$} \\
\hline \multicolumn{3}{|l|}{$\begin{array}{l}\text { Propicio ambientes en los cuales las niñas, niños y adolescentes se sientan seguros, útiles, } \\
\text { incluidos y reconocidos }\end{array}$} \\
\hline \multicolumn{3}{|l|}{$\begin{array}{l}\text { Permito que las niñas, niños y adolescentes a medida que crecen, vayan tomando } \\
\text { decisiones de acuerdo con sus capacidades para que adquieran seguridad y desarrollen su } \\
\text { autonomía }\end{array}$} \\
\hline \multicolumn{3}{|l|}{$\begin{array}{l}\text { Ofrezco las mismas oportunidades de socialización, juego, alimentación, conversación y } \\
\text { trato cariñoso a las niñas, niños y adolescentes que forman parte de mi fam ilia }\end{array}$} \\
\hline \multicolumn{3}{|l|}{$\begin{array}{l}\text { Dedico por lo menos } 15 \text { minutos a cada niña, niño y adolescente que forma parte de mi } \\
\text { familia, de manera independiente e individual, para desarrollar una actividad que ella o él } \\
\text { disfrute (ir al parque, leer cuentos, escuchar las canciones que le gustan, hacer dibujos, } \\
\text { salir a caminar, hacer actividad física, etc.) }\end{array}$} \\
\hline \multicolumn{3}{|l|}{ CON RESPECTO A LA INSTITUCIÓN EDUCATIVA } \\
\hline \multicolumn{3}{|l|}{ Asisto a los espacios de encuentro, tanto académico como culturales } \\
\hline \multicolumn{3}{|l|}{ Conozco el Manual de Convivencia de la Institución Educativa } \\
\hline \multicolumn{3}{|l|}{ Me involucro en los proyectos escolares } \\
\hline \multicolumn{3}{|l|}{$\begin{array}{l}\text { Me intereso tanto por sus procesos académicos como por aquellos de socialización y, } \\
\text { cuando identifico asuntos en los cuales la Institución Educativa puedo mejorar, lo } \\
\text { comunico de manera apropiada por medio de los conductos regulares establecidos }\end{array}$} \\
\hline \multicolumn{3}{|l|}{$\begin{array}{l}\text { Reportamos a la Institución Educativa situaciones de acoso escolar o de violencia escolar } \\
\text { en las que las niñas, niñas y adolescentes que forman parte de mi familia o sus compañeras } \\
\text { o compañeros estén de alguna manera involucrados }\end{array}$} \\
\hline Apoyamos y hacemos seguimiento a la Institución Educativa en el manejo de los casos & & \\
\hline lue nos bi & & \\
\hline
\end{tabular}

(a)

Tomado de la Guía 49 (Guías Pedagógicas de Convivencia Escolar) MEN.

Gracias por su apoyo y colaboración.

Figura 4. Encuesta aplicada a padres de familia

Fuente: tomada y adaptada de la guía 49. 
El taller diagnóstico fue otro de los instrumentos utilizados para identificar las necesidades de la población. En este sentido, el MEN (2013) los define como los que "se realizan usualmente para generar procesos de trabajo grupal enfocados en la formación, también pueden tener fines instructivos, consultivos o terapéuticos (p. 133). De esta manera, se utilizó e implementó en esta primera fase el taller a manera consultiva (ver figura 5). En general, se observaron faltas a las competencias ciudadanas comunicativas afectando altamente la convivencia escolar de las aulas

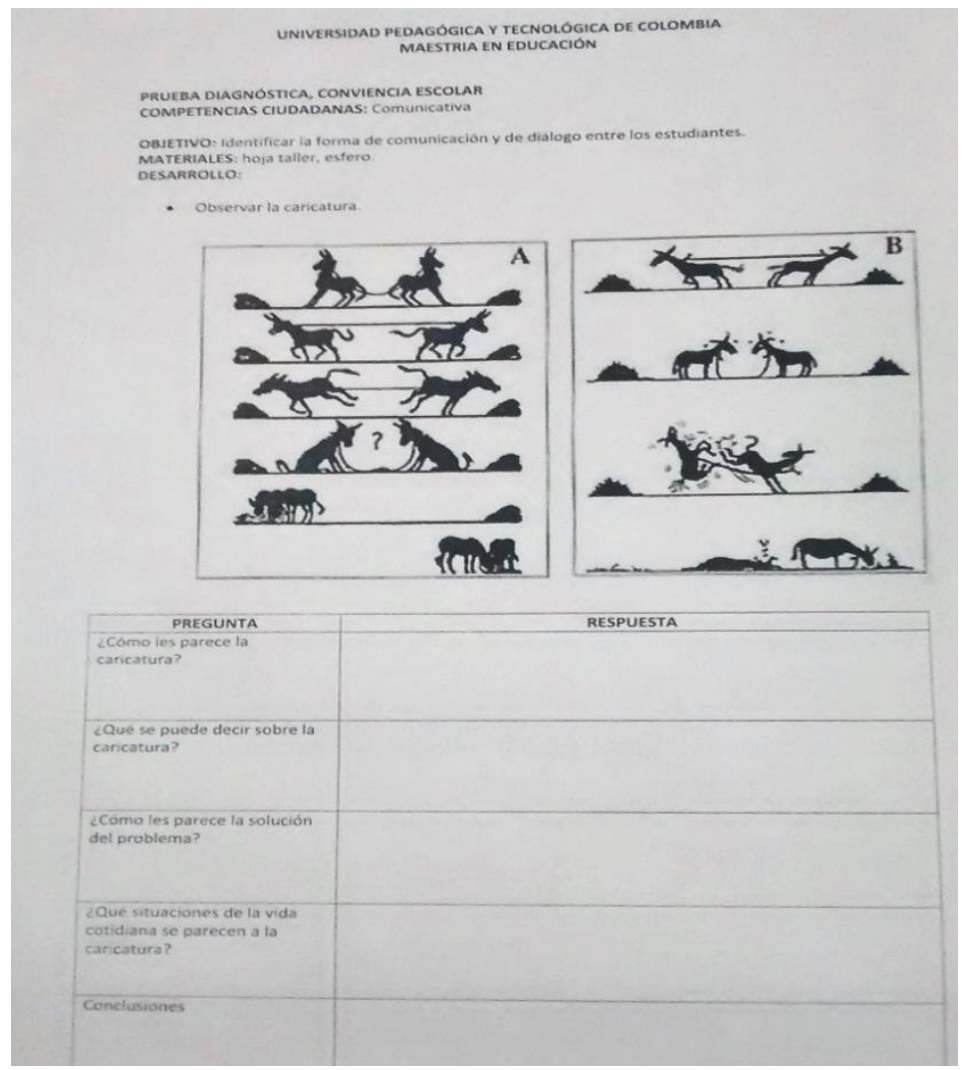

Figura 5. Taller diagnóstico implementado

Fuente: tomado y adaptado de - htpps//goo.glimages/1yMDLN Implementación: a partir del análisis de resultados del diagnóstico, se lograron identificar las dificultades en convivencia escolar y se procedió a la implementación de las estrategias pedagógicas a la luz de la Guía 49 del MEN, con el ánimo de promover dicha convivencia mediante las competencias ciudadanas comunicativas.

Es así como se establecieron: el diario pedagógico, juego de roles, talleres y pactos de aula como las estrategias culmen de la implementación. Las estrategias pedagógicas son necesidades específicas que permiten la participación activa y el vivir en comunidad (PérezJuste, 2007). 
El diario pedagógico: esta estrategia se conceptualiza como: "un instrumento que permite construir conocimientos a partir de una práctica intencionada, pues posibilita realizar análisis teóricos de la experiencia desde el registro detallado de su ejercicio" (MEN, 2013, p. 132). En este orden de ideas, la estrategia se logra establecer como una herramienta valiosa que permite sistematizar el ejercicio de la práctica docente.

A partir de la forma como se construyen conocimientos, también se envían mensajes democráticos y participativos. En este sentido, "en la medida en que se fortalezcan los canales de comunicación entre ambos, se garantiza una formación más pertinente, integral y justa" (MEN 2013, p. 198). Es así como dos elementos son claves en el aula para el fortalecimiento de la convivencia escolar: la iniciativa propia de las investigadoras para asumir el reto de trabajar a partir de las competencias ciudadanas comunicativas.

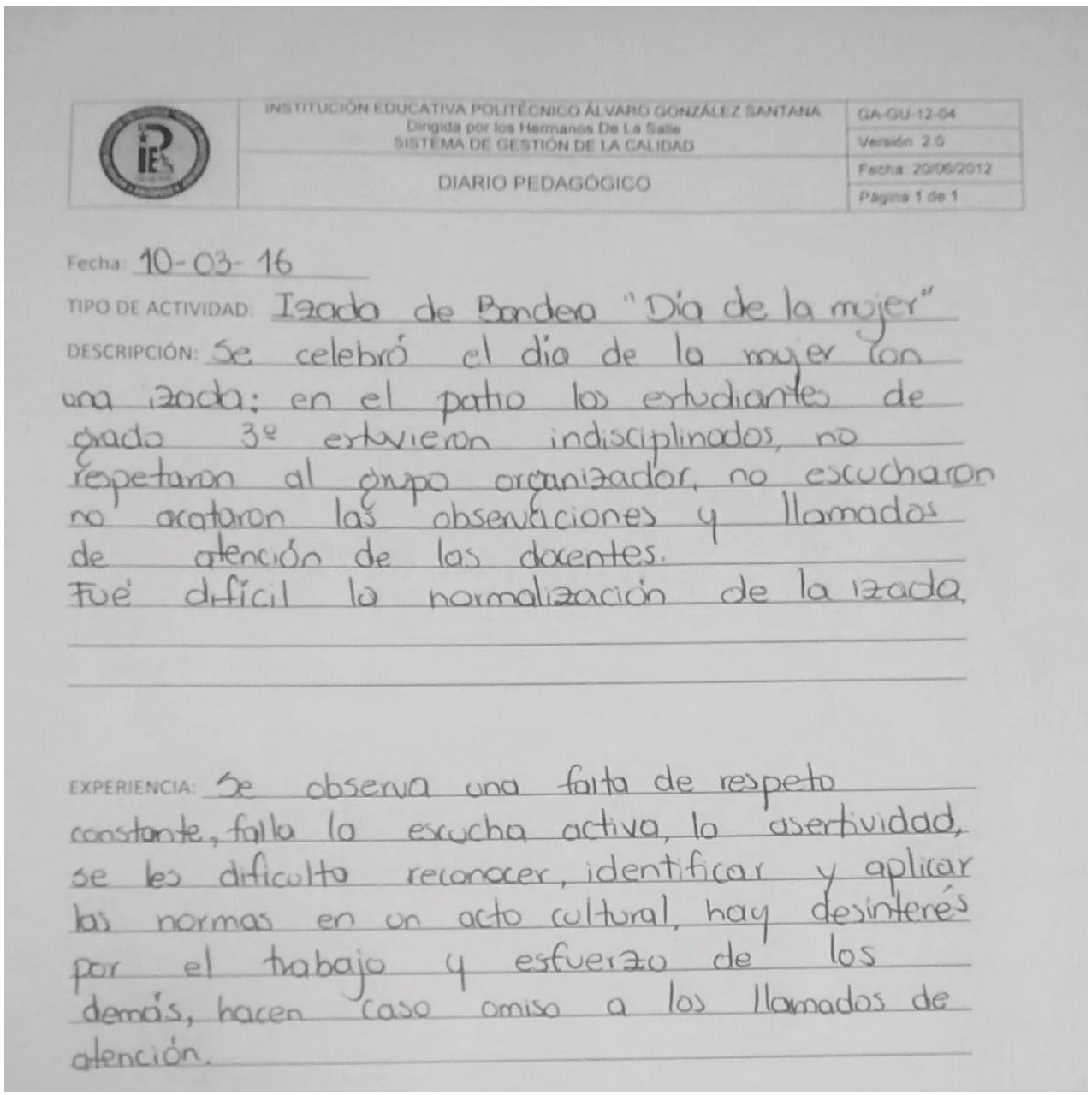

Figura 6. Diario pedagógico 2016.

Fuente: elaboración propia 
También trabajamos el juego de roles: el grupo de estudiantes se organiza en parejas para simular una situación de conflicto que sea similar a cualquiera de las que tienen de manera cotidiana, improvisando la manera cómo resolverían las diferencias. De esa manera pueden aprender y practicar competencias como la generación creativa de opciones, la toma de perspectiva, la escucha y la asertividad (Chaux et al., 2004).

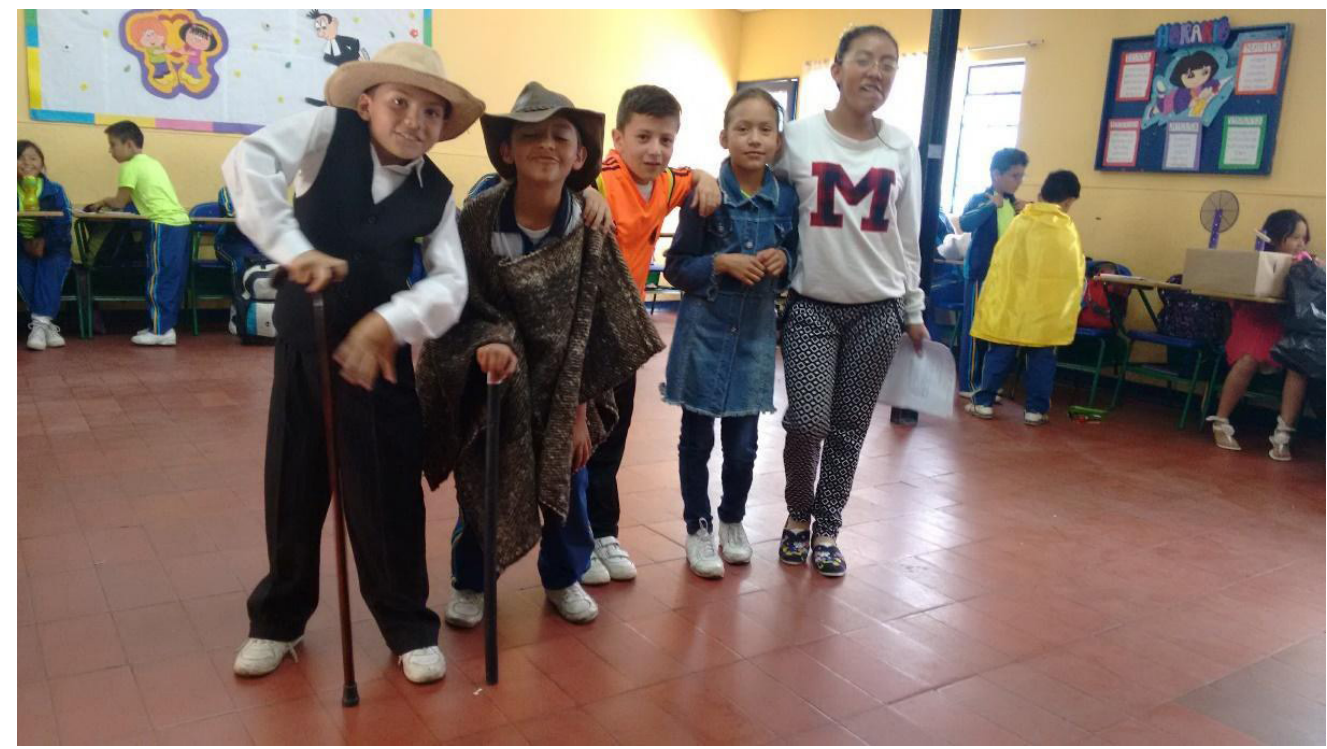

Figura 7. Fotografía juego de roles Fuente: archivo personal, 2016.
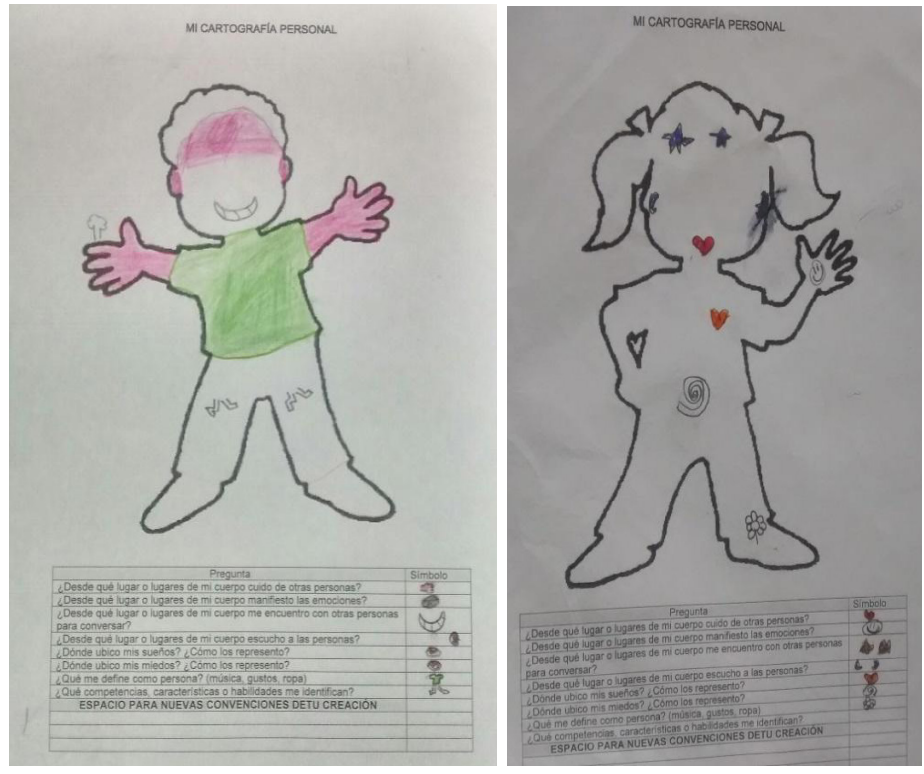

Figuras 8 y 9 . Fotografías taller aplicado Fuente: tomado y adaptado de la guía 49.
Pudimos ver que el juego de roles desarrolló las competencias ciudadanas comunicativas, a partir de la realidad representada, sin olvidar que la realidad depende del punto de vista del que observe, o desde donde se vivencie.

Los talleres se utilizaron como otra de las estrategias implementadas para el desarrollo de las competencias ciudadanas comunicativas. Los talleres se realizan usualmente para generar procesos de trabajo grupal enfocados en la formación, también pueden tener fines instructivos, consultivos o terapéuticos (MEN, 2013, p. 133). 
En los talleres, como estrategia pedagógica en el aula escolar, se trabajó el desarrollo de las competencias ciudadanas comunicativas mediante el desarrollo de diferentes ejercicios individuales y colectivos.

Finalmente, los pactos de aula que se definen como los acuerdos entre estudiantes y docentes para generar ambientes adecuados y aptos para la convivencia escolar. En este sentido se hace necesario comprender que el aula escolar es un hábitat de convivencia en el que emergen aspectos emocionales, conocimiento y roles de poder. De esta manera los pactos de aula pretendieron consolidar espacios de respeto y en los que las competencias ciudadanas adquirieron un verdadero sentido común en la cotidianidad y en la construcción de imaginarios colectivos.

En conclusión, la construcción concertada y colaborativa de los pactos de aula se reconoce como una oportunidad para desarrollar aprendizajes significativos entre miembros de la Institución Educativa y permite realizar el ejercicio de reconocer cuáles son mis derechos teniendo en cuenta el cumplimiento de los deberes.

\section{Evaluación de resultados: en} esta fase realizamos un análisis de la implementación de las estrategias en la que se lograron identificar las debilidades y fortalezas en la población objeto de estudio realizando una reflexión crítica. En este sentido, la evaluación es una constante durante el proceso investigativo desde la inmersión inicial, que para este caso es el diagnóstico y para la inmersión profunda que se ajusta a lo que para esta investigación fue la implementación de las estrategias pedagógicas (Hernández, Fernández y Baptista, 2006).

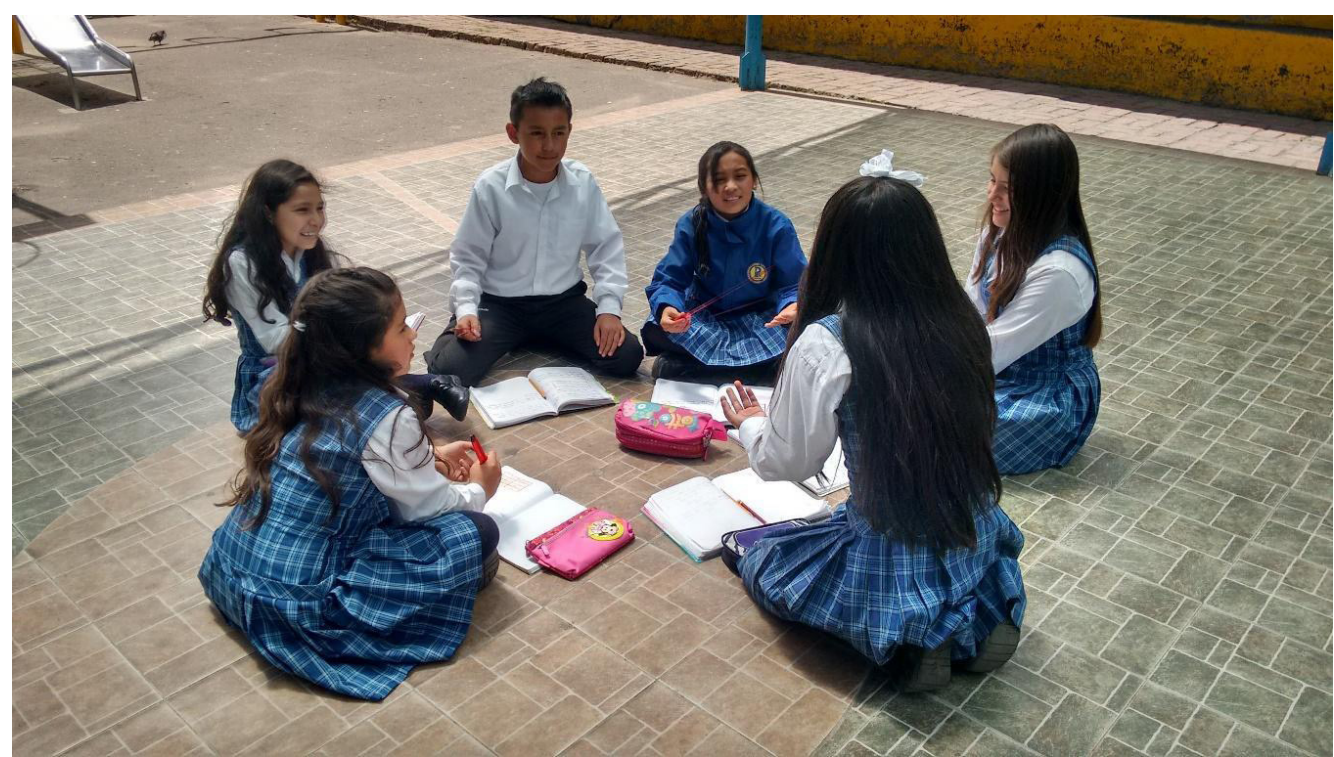

Figura 10. Fotografía pactos de aula.

Fuente: archivo personal.
Los pactos de aula que se definen como los acuerdos entre estudiantes y docentes para generar ambientes adecuados y aptos para la convivencia escolar. 


\section{En el grado tercero}

De acuerdo conlo anterior, comenzamos la presentación de resultados del grado tercero. Un primer elemento en el que intervienen los distintos actores de la comunidad educativa, es el clima de aula, esta subcategoría de "Convivencia y paz" (Estándares de Competencias ciudadanas, MEN, Guía $\mathrm{N}^{\circ} 6$, 2004) representa y tiene un valor muy importante en el desarrollo de competencias ciudadanas, pues debe tener una práctica docente con características democráticas, como lo habíamos citado anteriormente. Asumir el reto de formar estudiantes a partir de las competencias ciudadanas comunicativas, nos llevó a pensar y reflexionar en la forma como estamos ejerciendo nuestra práctica docente en el aula para transformar y construir una distinta que, en consecuencia, aporte al objetivo que nos llevó a plantearnos este reto: el de fortalecer la convivencia escolar a partir del desarrollo de las competencias ciudadanas comunicativas.

En este sentido, para la competencia comunicativa: asertividad, se identificaron características comunes en el grupo y otras en cambio de tipo individual pero que de forma simultánea, aportan al fortalecimiento de la convivencia escolar y que a lo largo de esta evaluación iremos exponiendo. De acuerdo con esto, la relación del docente en el aula comenzó a generar una perspectiva en los estudiantes frente a la asertividad. Si el docente transmite sus ideas de forma adecuada y sin herir a sus estudiantes, y utilizando un vocabulario pertinente, lo mismo hace el estudiante en el momento de comunicarse con los demás. El estilo docente marcó la calidad de las relaciones entre las mismas docentes y los estudiantes. En este sentido, dichas relaciones se marcaron a partir de la forma como nos lográbamos comunicar con ellos, manteniendo relaciones, dialogicidad, la mirada atenta a realidades, a problemáticas emergentes, posibilitando la fusión de investigación y educación para renovar el re-encuentro entre maestros y estudiantes (Orrego \& Toro, 2014), encaminándolos hacia el desarrollo de la asertividad.

Aunado a esto, identificamos la argumentación como un segundo elemento de las CCC. Esta competencia, que hace referencia a la capacidad de expresar ideas de forma clara y con criterio, se trabajó para su desarrollo en el aula en las diferentes asignaturas de forma transversal y con las estrategias pedagógicas mencionadas, que dejaron resultados significativos y medibles en el desempeño académico y en la construcción colectiva del clima de aula que, finalmente, hace sinergia en el fortalecimiento de la convivencia escolar.

La capacidad de argumentación se incrementó en casi la mitad de los estudiantes. En este resultado, es importante analizar el principio pedagógico. Además, otro de los elementos valiosos que se rescata en el desarrollo de esta competencia y como se mencionaba en el párrafo anterior, es la capacidad de diálogo que lograron 
los estudiantes. Definitivamente, consolidaron esta expresión como una competencia comunicativa en la mayoría de los estudiantes. Esta característica comenzó su desarrollo a partir del mismo estilo docente, que a diferencia del tradicionalista, permite la utilización de esta herramienta comunicativa como una forma de solucionar conflictos entre las relaciones maestro-estudiante y estudiante-estudiante. Para lograr este fin, además de las estrategias mencionadas en varias ocasiones, se aplicaron otras herramientas que permitieron la construcción de escenarios de diálogo.

Por otra parte, en cuanto a la escucha activa en los estudiantes de grado tercero, mediante la implementación de las estrategias mencionadas en el aparte anterior, se pudo identificar que la mayoría de ellos lograron mejorar su habilidad de escucha y este hecho se reflejó en la forma de expresar las ideas de los demás: es decir, estos niños $\mathrm{y}$ niñas adquirieron un argumento con mayor solidez y veracidad para dar sus opiniones y participar activamente en clase, mejoraron las conductas tímidas y de inseguridad que eran cotidianas; estas características se reflejaron en el incremento de la participación en clase y en las diferentes actividades que se desarrollan en la IE. Mejoraron notoriamente su capacidad de parafraseo: normalmente los estudiantes dudaban y de hecho les costaba expresar sus ideas a partir de una lectura o de una exposición, por ejemplo, luego de la implementación de las estrategias pedagógicas lograron expresar ideas con sus propias palabras a partir de lo que escuchaban. De esta manera, los niveles de confianza en ellos mismos, la autonomía y la asertividad se desarrollaron $y$ fue un evento consecuente para la facilitación de las actividades académicas y, por supuesto, de la convivencia escolar. El manual de convivencia de la Institución como componente del PEI institucional, fue asimilado y llevado a la praxis por los niños con mayor empatía, pues ya no se ve como una "cartilla de obligaciones" sino como una carta de navegación de cuidado que permite su verdadera esencia "saber convivir".

\section{En el grado cuarto}

Se afianzó en los estudiantes, la capacidad para defender sus derechos y opiniones sin ocasionar daño a los demás, para hacer críticas de manera constructiva y también a que reconocieran sus propios errores. De dicha aplicación, fueron encontrados los siguientes resultados.

Las habilidades de asertividad se fueron desarrollando progresivamente, la mayoría de estudiantes empezaron por entender la importancia de la comunicación; luego, poco a poco, se fue evidenciando -la capacidad- que desarrollaron para expresar sentimientos en un ambiente de respeto, fueron capaces de tomar decisiones sin miedo al rechazo, expusieron sus puntos de vista sin temor a que sus opiniones no fueran iguales a los demás, demostraron confianza en sí mismos, afrontaron los conflictos de manera más serena.

Cabe señalar que, después de implementar las cuatro estrategias propuestas y otras como charla con
Las habilidades de asertividad se fueron desarrollando progresivamente, la mayoría de estudiantes empezaron por entender la importancia de la comunicación 
Es importante resaltar que, para lograr en los estudiantes una comunicación asertiva, fue muy relevante el estilo docente, porque en el aula escolar los niños se encontraron con docentes asertivo/democráticos el psicoorientador, conversatorios $\mathrm{y}$ debates sobre el tema, se obtuvieron resultados favorables en la mayoría de los estudiantes, logrando buena capacidad de comunicación y relaciones sociales (Ospina, 2014). Al mismo tiempo, una minoría de estudiantes no logró desarrollar esta habilidad, en ellos se encontraron características como timidez, se sentían incomprendidos, con sentimientos de frustración, situación por la cual fue difícil el mejoramiento de las habilidades comunicativas en este grupo.

Es importante resaltar que, para lograr en los estudiantes una comunicación asertiva, fue muy relevante el estilo docente, porque en el aula escolar los niños se encontraron con docentes asertivo/democráticos, como lo contempla el manual de convivencia de la IEPAGS en lo concerniente a deberes del docente: "Mantener comunicación asertiva con compañeros, padres, acudientes, estudiantes, y demás integrantes de la comunidad educativa en una forma respetuosa y amable". Esto permitió que los estudiantes se sintieran en un ambiente de confianza y seguridad para ir avanzando en el desarrollo de dichas competencias.

Finalmente, se detectó que la competencia de asertividad en el grado cuarto permitió un mejoramiento en la autoestima de los estudiantes, se fortalecieron las relaciones interpersonales y la convivencia escolar.

En la competencia de argumentación, se observó mayor desarrollo en lo que tenía con ver con el trabajo en grupo, pues allí los estudiantes se encontraron con actividades en las que tenían que ser responsables individualmente para cumplir con los objetivos grupales, no debían únicamente expresar la opinión sino encontrar la mejor manera de explicar sus puntos de vista, interiorizando que su intervención beneficiaba o afectaba al colectivo. Es así como vemos que al afianzar las CCC, se va dando también el fortalecimiento de la Convivencia Escolar.

Así mismo, se pudo evidenciar la importancia de utilizar la argumentación en el desarrollo de las clases, pues esta trae consigo espacios de participación donde el estudiante se sintió miembro activo dentro de su proceso de formación y no se limitó solamente a recibir ideas de los demás, sino que sus aportes son explicados por medio de argumentos que forjaron procesos de pensamiento como el análisis, la reflexión y una postura crítica.

En cuanto a la escucha activa, se encontró en los estudiantes timidez al momento de expresarse en público, $\mathrm{o}$ de replicar las intervenciones $\mathrm{O}$ exposiciones de sus compañeros; pero, con el paso del tiempo, fueron aplicando las técnicas de escucha activa y acogieron la oportunidad que estas le ofrecen para mejor el proceso de comunicación.

Al mismo tiempo, fue indispensable el estilo docente, teniendo en cuenta que el maestro, en muchos casos, es el ejemplo a seguir especialmente por los niños (as), por lo tanto este debe demostrar una actitud positiva al momento de escuchar a los estudiantes, afianzar los buenos hábitos de escucha, 
crear ambiente de respeto hacia el uso de las palabra donde se favorece el diálogo libre y voluntario, conseguir que los estudiantes sostengan discusiones y respeten el turno para hablar, es así como nuevamente observamos que el modelo pedagógico tuvo un papel muy importante en el desarrollo de dicha competencia (Samaca, 2016).

Para finalizar, es necesario señalar otras características encontradas en los estudiantes para el fortalecimiento de las CCC, entre ellas están el enriquecimiento del vocabulario, la capacidad de parafrasear, mejoramiento de la capacidad para expresarse en público, participación voluntaria en actividades, fortalecimiento del trabajo colaborativo enfatizando en los aportes individuales para lograr los objetivo comunes. De esta forma, se logró establecer relaciones más sólidas y construir acuerdos que fortalecieron la convivencia escolar.

\section{En el grado quinto}

Después de aplicar los talleres, los conversatorios, los juegos, diálogos, entre otros; se pudo observar una mejora en la asertividad de más de la mitad del curso, el proceso fue progresivo en su fortalecimiento, puesto que desde la observación, la aplicación de los primeros talleres, conversatorios y juegos; se evidenciaba el querer imponer la voluntad de la gran mayoría, las ofensas entre sí y algunas agresiones eran muy comunes. Con el pasar del tiempo, se fueron concientizando de su papel en el desarrollo de las actividades, observaban la participación de los demás y reflexionaron en la transformación de sus actitudes, mejorando así la capacidad para expresar sus sentimientos, emociones, necesidades, intereses, opiniones; hacer valer sus derechos, todo esto de una manera clara, respetuosa y democrática. Con todo y lo anterior, cabe señalar que la Institución Educativa ha trabajado un estilo docente democrático asertivo donde se establecen normas, reconocen y respetan la individualidad, se toman decisiones de forma conjunta teniendo en cuenta el bien común, no el individual.

Ahora bien, en cuanto a la competencia de argumentación, se fortaleció la capacidad de defenderideas, exponiendo sus razones de una forma coherente y respetuosa sin querer ser impositivos, o afectando las de los demás; fue un poco más complejo, ya que los estudiantes pretendían tener la razón sin dar sus argumentos válidos, simplemente imponiéndolos y reaccionando con enojo si no se tenían en cuenta los suyos; en el grado quinto, por su edad cognitiva y sus procesos mentales más avanzados, tenían una capacidad argumentativa buena, algunos estudiantes más que otros, pero por lo mismo era necesario encaminar la competencia hacia todos los estudiantes para lograr el objetivo.

Sin embargo, después de la aplicación de las estrategias, el resultado que se obtuvo fue una mejora de más de la mitad de los estudiantes, conforme se fue aplicando el proceso, los estudiantes más apáticos empezaron su mejora al exponer las ideas en sus intervenciones, cabe aclarar que, para esta competencia, es necesario tener un vocabulario más amplio y eso 
se consigue con la lectura; por dicha razón, la investigación fue transversal, se reforzó y se sigue reforzando en todas las áreas del conocimiento y sumado a los proyectos de pastoral que trabaja La Salle.

Por otra parte, al analizar la competencia de escucha activa, se presentaron ciertas dificultades, puesto que hacía falta más concentración y atención al escuchar a los demás, se debía volvera repetirla actividad una y otra vez, sin obtener los resultados esperados. Se observó que a esta edad, la tecnología ha acaparado la atención de los estudiantes, generando deficiencias en la escucha, siendo esta fundamental en las relaciones interpersonales y en el proceso comunicativo. Un estudiante que es escuchado, se siente reconocido; para que la escucha activa fuera adecuada, se necesitaba recalcar en el lenguaje no solo verbal, sino corporal, el lenguaje del cuerpo y las posturas, se retomaron: contacto visual, tono de vOz adecuada, receptividad; en fin, estos elementos se hicieron indispensables en el fortalecimiento de la CCC, de una forma paulatina y constante para lograr su mejora, así se pudo favorecer el ambiente democrático y participativo que nos señala el modelo pedagógico institucional.

Para concluir, el grado 507 tuvo una mejora notable en más de la mitad del curso, debido al constante trabajo y aplicación de las estrategias, la mayoría de estudiantes son reconocidos en la actualidad, por su buen rendimiento académico y relaciones interpersonales, la forma de solucionar dificultades, la participación y expresión, la argumentación y escucha frente a los demás compañeros de otras sedes que conforman los grados sexto, alcanzando los objetivos propuestos para esta investigación.

\section{Conclusiones}

Las Instituciones Educativas son el entorno en que los estudiantes se preparan para su vinculación al mundo competitivo, pero también es el lugar donde se construyen las primeras relaciones sociales después de la familia, es en el colegio donde se forma en varios aspectos que les permitirá a los estudiantes desarrollarse en un mundo cambiante con autonomía e independencia. Al trabajar en convivencia escolar, desarrollando las competencias ciudadanas comunicativas, en la sede Campoamor se fortalecieron las diferentes relaciones que se construyen a diario desde las aulas escolares propiciando un clima adecuado para desenvolverse como seres humanos capaces de transformar su propio contexto, mediante la aplicación de estrategias que conllevan a la comunicación asertiva, la argumentación y la escucha activa, los cuales son indispensables para convivir en un ambiente armónico donde siempre que se presente un desacuerdo se solucione poniendo en práctica lo aprendido, ahora bien, todo esto es un aporte a la comunidad educativa y un inicio para proponer esta investigación como pilar fundamental de la construcción de un mundo mejor.

En este sentido, la escuela se ve afectada por conductas que aquejan la convivencia escolar como los problemas de disciplina, la violencia escolar, la falta de argumentación, el no 
saber escuchar al otro y el proceso de comunicación asertiva. Cabe señalar que la convivencia escolar en las instituciones educativas, requiere mayor atención con relación a la que actualmente merece; es un motor indispensable en la construcción de nuevos imaginarios colectivos tendientes a la formación de ciudadanos útiles y reflexivos acordes al proceso de transformación que se viene entretejiendo en lo local, regional y nacional.

Partiendo de esto, la convivencia escolar con un modelaje acertado, acorde y franco, logra desarrollar estructuras y esquemas que facilitan el aprendizaje en el aula y, por supuesto, mejora el clima de aula escolar.

El desarrollo de competencias ciudadanas comunicativas, por su parte, logran construir en el aula dinámicas pertinentes en el sentido de generar una real escucha, la capacidad de expresar ideas y sentimientos sin sentir temor o miedo, y la capacidad de exponer puntos de vista claros y oportunos. Estas competencias, definitivamente, son transversales a la cotidianidad escolar, puesto que fortalecen no solo en el campo educativo sino el ámbito personal, por ende, el maestro de aula comienza este camino de construcción ciudadana a partir de una práctica reflexiva que le permita identificar su realidad en el aula escolar, sus propios miedos, sus propios errores para revolucionarlos y apostarlos a un cambio esquemático de costumbres $\mathrm{y}$ tradiciones que, en consecuencia, se reflejan en nuevas competencias de los estudiantes que tiene la grata y no simple labor de educar. La didáctica, este elemento propio y soporte de una práctica pertinente para el desarrollo de competencias ciudadanas comunicativas, es sin duda alguna -hablando desde la experiencia- un factor clave y esencial; esta permite la inclusión y la interiorización profunda de habilidades y actitudes acordes y fructíferas en la convivencia escolar.

La co-relación entre el maestro y el estudiante, este lenguaje oculto logra desarrollar en el estudiante características positivas $\mathrm{y} / \mathrm{o}$ negativas dependiendo de la madurez profesional con la cual se asuma. El maestro al llegar al aula, debe ponerse su armadura de madurez personal y profesional, y concebir ese espacio como uno sagrado, no en vano son vidas enteras las que se están formando y moldeando. Cada palabra, cada actitud del maestro, es interiorizada por el niño o la niña y repercute en su construcción personal y social.

De manera que, la educación no pierde su papel fundamental, histórico y crucial para la construcción de un tejido social diferente. Es un proceso lento, complejo a veces, pero esperanzador donde esta herramienta apuesta hacia la generación de una ciudadanía plena, que evoluciona, es decir, que es capaz de solucionar los conflictos a los que se vea enfrentado de una forma pacífica. Cada maestro en el aula, además de impartir conocimientos y cumplir con metas, estándares de pruebas nacionales e internacionales, acortando este proceso a una fábrica de conocimiento en la que se genera un producto, tiene el privilegio de aportar -aunque pareciera poco- para que esta utopía aterrice como un gran acierto a la construcción de una Colombia diferente, estable y duradera.
La co-relación entre el maestro y el estudiante, este lenguaje oculto logra desarrollar en el estudiante características positivas $\mathrm{y} / \mathrm{o}$ negativas dependiendo de la madurez profesional con la cual se asuma. 


\section{Referencias}

Bonilla, E., \& Rodríguez, P. (2005). Más allá del dilema de los métodos. Colombia: Editorial Nomos S.A.

Chaux, E. Lleras, J., \& Velásquez, A.M. (Eds.) (2004). Competencias ciudadanas: de los Estándares al aula. Bogotá: Uniandes.

Dubet, F., \& Martuchelli, D. (1998) En la escuela. Sociología de la experiencia escolar. Revista Mexicana de Investigación Educativa. Recuperado de http:/ / www.redalyc.org/articulo.oa?id=14001008

Elliott J. (1990). La investigación-acción en educación. Ediciones Morata.

Hernández, R., Fernández, C., \& Baptista, P. (2006). Metodología de la investigación (4a. ed.) México, Iztapalapa: McGraw Hill.

Institución Educativa Politécnico Álvaro González Santana. (2014). Proyecto educativo Institucional (PEI).

Institución Educativa Politécnico Álvaro González Santana. (2015). Manual de Convivencia.

MEN. (2004). Formar para la ciudadanía jSí es posible! Guía Nº 6. Bogotá: IPSA.

MEN. (2013). Guías Pedagógicas para la convivencia escolar. Guía No 49. Bogotá: Amado Impresores.

Orrego Noreña, J., \& Toro González, L. (2014). Relaciones vitales: el aula como escenario permanente de investigación. Praxis \& Saber, 5(10), 121 - 139. https://doi.org/10.19053/22160159.3025

Ospina Nieto, Y. (2014). Rescatar lo antropológico... una necesidad de la educación. Praxis \& Saber, 5(10), 193 - 218. https://doi.org/10.19053/22160159.3029

Pérez, R. (2007). Educación, ciudadanía y convivencia. Diversidad y sentido social de la educación. Revista de Pedagogía, 59(2-3), 239-260.

Samacá Bohórquez, Isabel. (2016). El espíritu científico en la primera infancia. Praxis \& Saber, 7(13), 89-106. https://doi.org/10.19053/22160159.4167 